\title{
Biocompatibility Evaluation of a New Hydrogel Dressing Based on Polyvinylpyrrolidone/Polyethylene Glycol
}

\author{
Esmaeil Biazar, ${ }^{1}$ Ziba Roveimiab, ${ }^{2}$ Gholamreza Shahhosseini, ${ }^{3}$ \\ Mohammadreza Khataminezhad, ${ }^{4}$ Mandana Zafari, ${ }^{5}$ and Ali Majdi ${ }^{5}$ \\ ${ }^{1}$ Department of Chemistry, Tonekabon branch, Islamic Azad University, Tonekabon, Iran \\ ${ }^{2}$ Young Researchers Club, Science and Research Branch, Islamic Azad University, Tehran, Iran \\ ${ }^{3}$ Nuclear Agriculture Research Department, Agricultural, Medical and Industrial Research School, \\ Nuclear Science and Technology Institute, Iran \\ ${ }^{4}$ Department of Microbiology, Tonekabon branch, Islamic Azad University, Tonekabon, Iran \\ ${ }^{5}$ Young Researchers Club, Tonekabon Branch, Islamic Azad University, Tonekabon, Iran
}

Correspondence should be addressed to Esmaeil Biazar, e.biazar@tonekaboniau.ac.ir

Received 27 March 2011; Revised 22 June 2011; Accepted 23 June 2011

Academic Editor: Masa-Aki Shibata

Copyright ( 2012 Esmaeil Biazar et al. This is an open access article distributed under the Creative Commons Attribution License, which permits unrestricted use, distribution, and reproduction in any medium, provided the original work is properly cited.

\begin{abstract}
The composition of the dressings is based on polyvinylpyrrolidone (PVP), polyethylene glycol (PEG), and agar. The electron beam irradiation technique has been used to prepare hydrogel wound dressings. The in vitro biocompatibility of the hydrogel was investigated by check samples (hydrocolloid Comfeel), antibacterial test (Staphylococcus aureus, Staphylococcus epidermidis, Pseudomonas aeruginosa, Escherichia Coli k12), anti fungal test (Candida Albicans) and cytotoxicity test (Fibroblast L929). Results have shown cell attachment characteristics and nontoxicity of all samples. Antibacterial testing also showed that the antibacterial effect of the hydrogel sample to the check sample increased to $30 \%$. Also, investigation of antifungal analysis did not show any trace of fungi growth on the surface of the hydrogel, whereas antifungal effect did not observe on the surface of the check sample. Finally, this hydrogel sample showed a good in vitro biocompatibility.
\end{abstract}

\section{Introduction}

Bed sore is considered a wound resulted due to exerting high and protracted capillary pressures on the surface of the skin long time and can lead to the necrosis of the soft tissues $[1,2]$. Among of these necrotic areas the pressure ulcers, burnt ulcers, as well as diabetic wounds can be mentioned $[3,4]$. One way for healing these wounds is using of dressings. Conventional dressings like gauze and cotton have been increasingly applied. Modern dressing has been used in the past two decades provided the humid healing of the wounds [5]. They have possessed most of the characteristics of an ideal dressing. Transparent dressing [6], hydrogel [7], alginate [8], and foam and hydrocolloids [9] are some examples of dressing for wound healing. Hydrogels are provided in two shape planes and shapeless gels. Hydrogels contain large amounts of water and the jelly substance constructed the polymer network $[10,11]$. Other examples of hydrogels are polyethylene oxide or polyvinyl pyrrolidone, carboxyl methyl cellulose, alginate, collagen, and other materials [11]. Also, PEG and PVP are hydrogel that can be used as wound dressing [12].

In vitro biocompatibility tests of dressings include cytotoxicity and antimicrobial tests (antibacterial and antifungal). Also, in vivo tests include irritation, sensitization, implantation, acute and chronic toxicity, and systemic toxicity. Fungi and bacteria are the resistant factors for fast wound healing. In our previous work [13], the application of the electron accelerator (Rhodotron TT200) for preparation of hydrogel dressings with polyvinyl pyrrolidone, polyethylene glycol, agar, and water composition was investigated. The effect of some parameters such as gel fraction and maximum swelling on the properties of the dressing demonstrated that hydrogel has the proper physical and mechanical properties. In this research, in vitro biocompatibility of hydrogel samples has been compared with the hydrocolloid (Coloplast Ltd. 


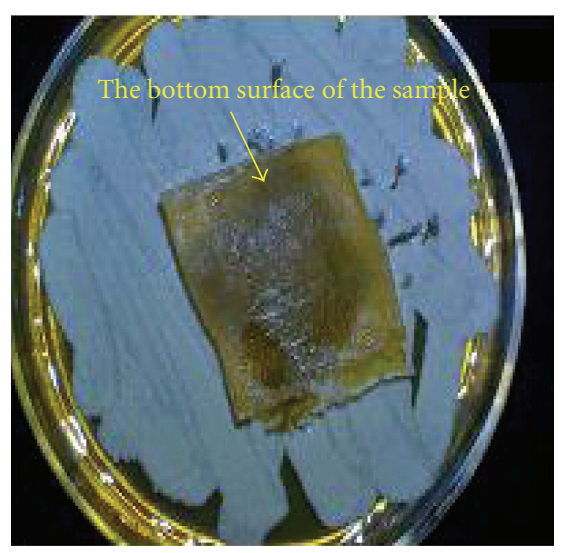

(a)

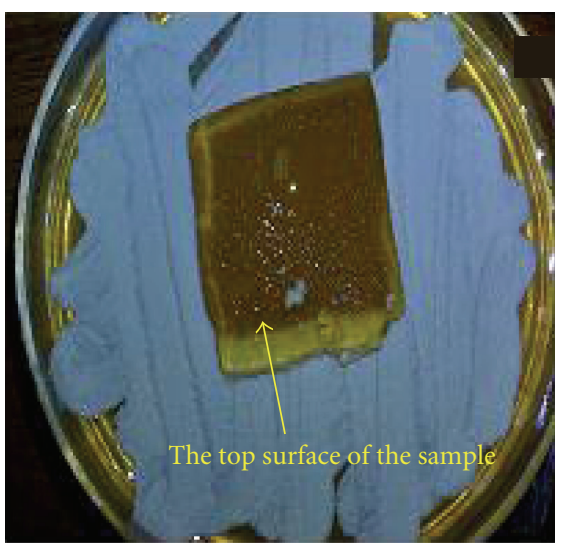

(b)

Figure 1: Hydrogel sample: (a) The bottom surface of the sample no growth was detected; (b) The top surface which showed the lack of full growth of fungi.

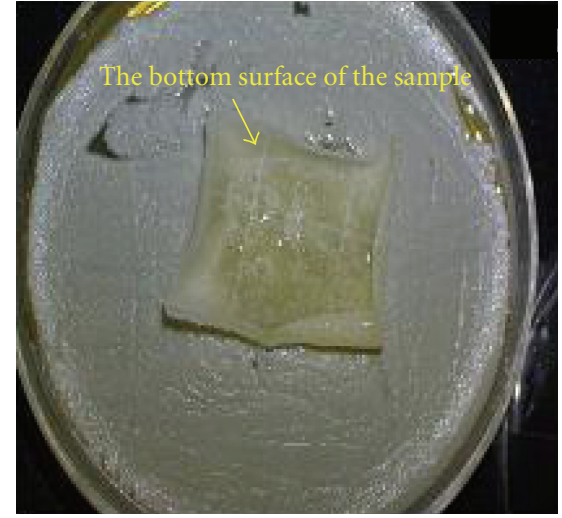

(a)

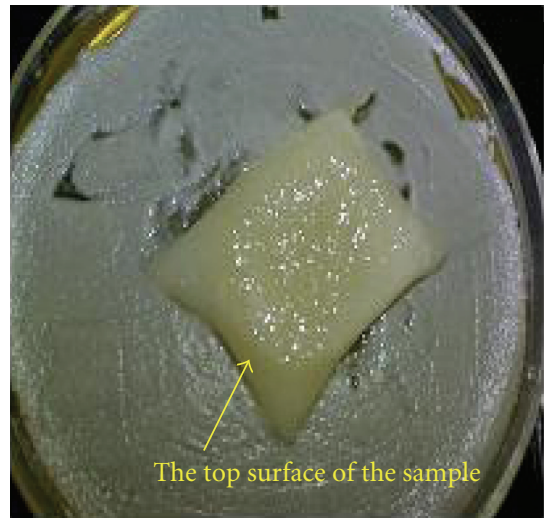

(b)

Figure 2: Transparent Comfeel sample: (a) The bottom surface which showed the growth of fungi; (b) The top surface where fungi has completely grown on it.

Comfeel plus Hydrocolloid dressing, England) as check and control sample through standard cytotoxicity (ISO 10993), antibacterial, and antifungal tests.

\section{Experimental}

2.1. Materials. The hydrogel samples (PEG, PVP, agar, and water) have been prepared by the Radiation Processing Center in Yazd as follows [13]. PVP (BASF, MW 1/4, 90000), PEG (BASF, MW 1/4, 200), agar (Difco) and water have been used to prepare the hydrogel. First, an aqueous solution of these materials has been prepared, and then, a homogeneous solution has been formed by solving and mixing the materials in a constant temperature. The solutions have been formed as wound dressings in the molds. After cooling down the solution, a gel structure with high viscosity has been manufactured. Gel samples are irradiated under a proper dose $(600 \mathrm{kGy} / \mathrm{min})$ and radiation energy of $10 \mathrm{MeV}$ in Electron Accelerator for crosslinking. Finally, the samples have been sterilized under an appropriate dose of radiation. To assess the cytotoxicity of hydrogel samples, check sample (Comfeel: hydrocolloids contain CMC and calcium alginate; NHS: ELM351 Coloplast, Britain ltd.), polystyrene control (TCPS) and fibroblast cells (L929) were used. The Staphylococcus epidermidis, Staphylococcus aureus, Pseudomonas aeruginosa, Escherichia coli bacteria (Iran Pastor Institute), and Candida albicans fungi (Iran Pastor Institute) were used to assess antimicrobial effect.

2.2. Antifungal Analysis. A cell suspension was prepared by C. albicans and 8-10 cc physiologic serum in hemolysis tube. 64230 cells per each microliter of the suspension were put on the neobar lam. Then, a part of this suspension was poured into the petridish and cultured on the Sabroe Dextrose agar (SDA) with $0.05 \%$ chloramphenicol. The wound dressings of Comfeel and the hydrogel samples (20 samples with the best physical properties) were cut out $(1.5 \times 1.5 \mathrm{~cm})$ and placed on the culture media. C. albicans was placed on the (SDA) media along with dressing in the incubator at $35^{\circ} \mathrm{C}$ for $3-5$ days. 


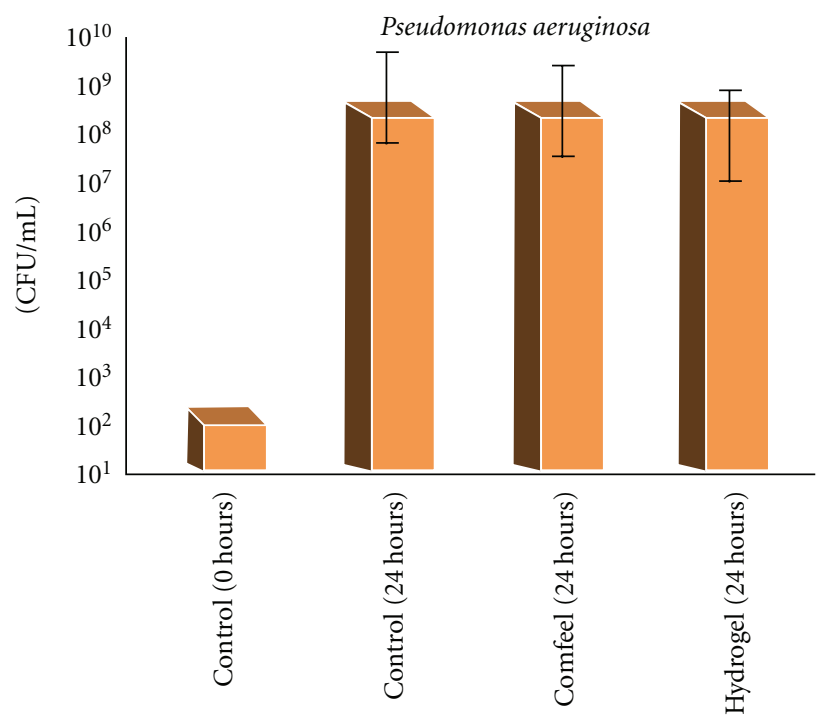

(a)

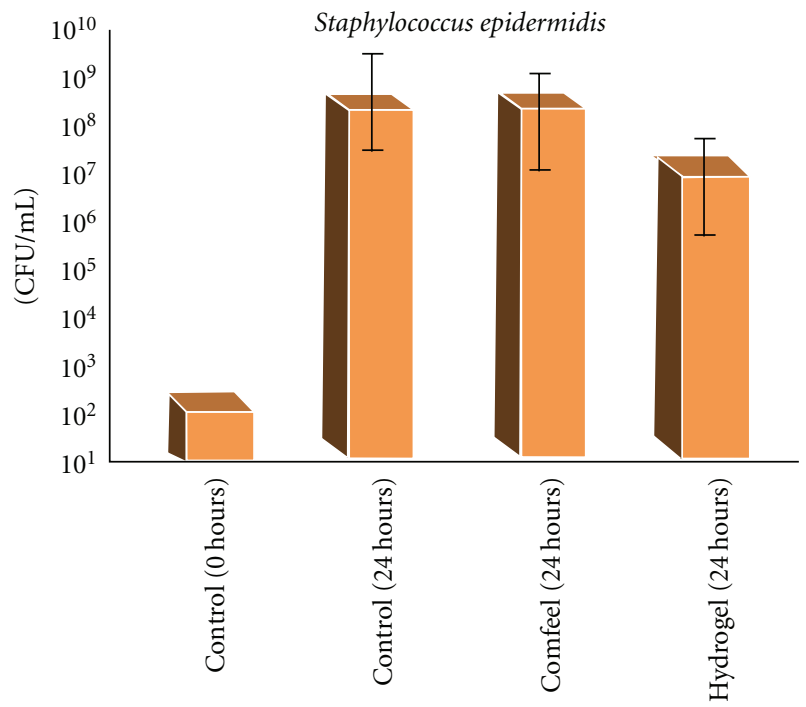

(c)

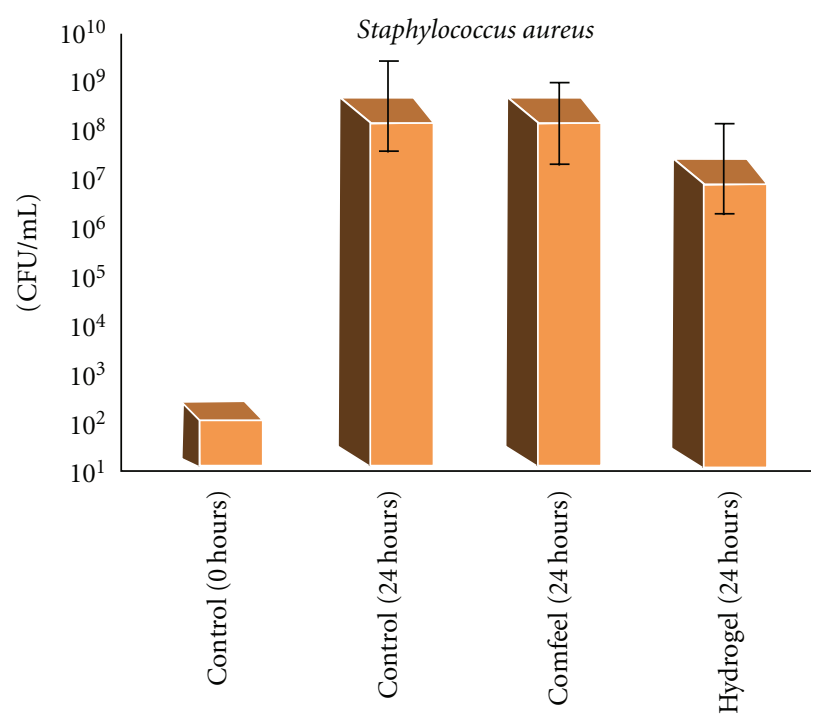

(b)

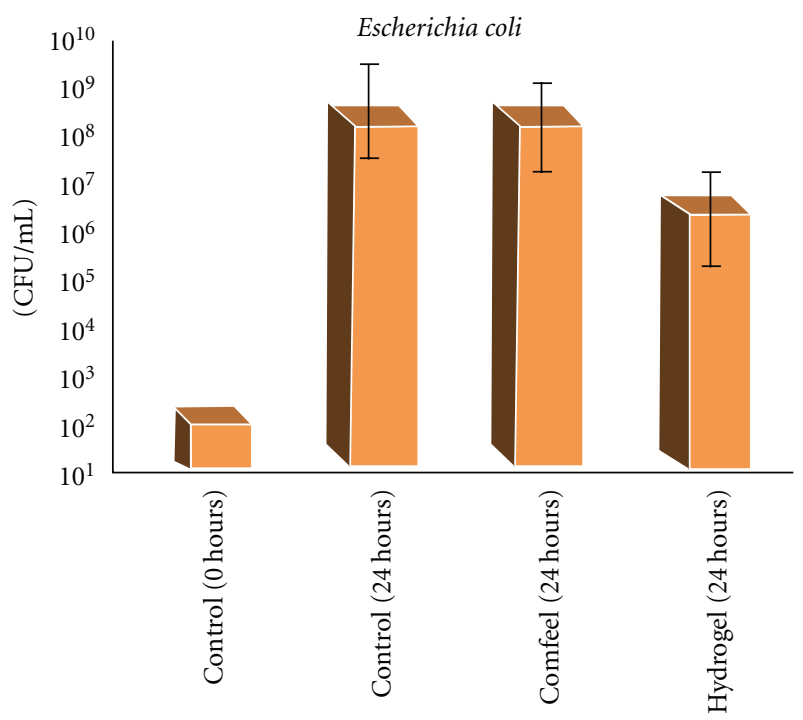

(d)

FigURE 3: The number of microorganisms, control (a) after 0 hours, control (b) after 24 hours, comfeel (c) after 24 hours, and hydrogel (d) after 24 hours.

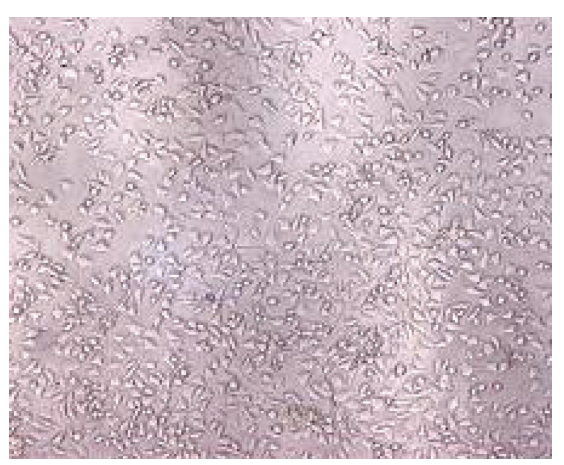

(a)

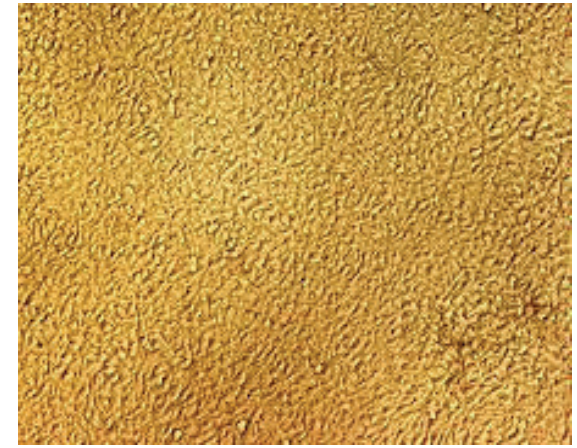

(b)

FIgURE 4: Fibroblast cells growth on the control sample (TCPS) (100×): (a) After 24 hours; (b) After 48 hours. 


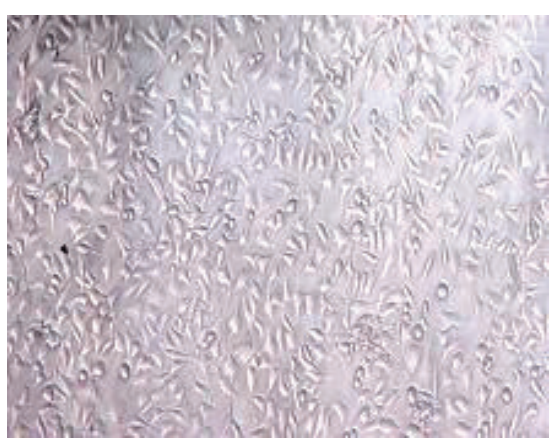

(a)

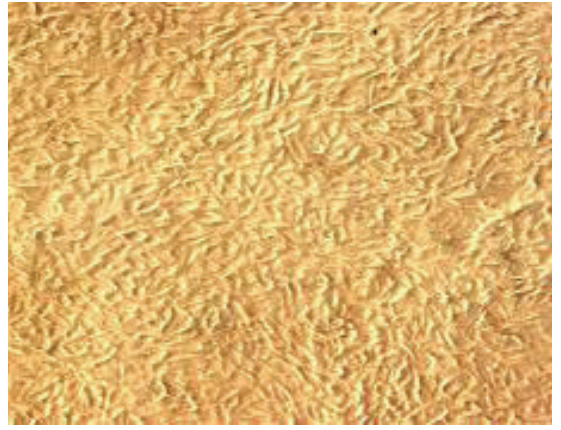

(b)

Figure 5: Fibroblast cells growth on the (Comfeel) control sample (100×): (a) After 24 hours; (b) After 48 hours.

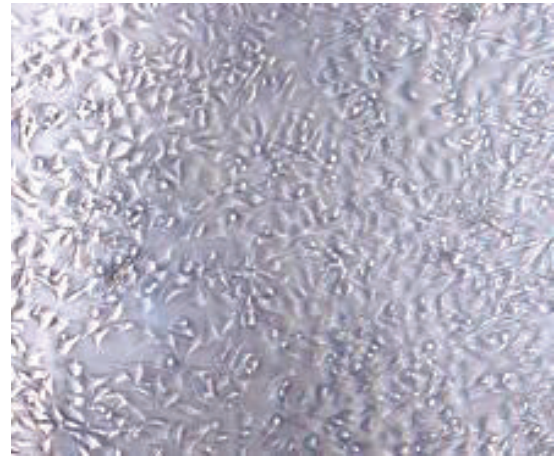

(a)

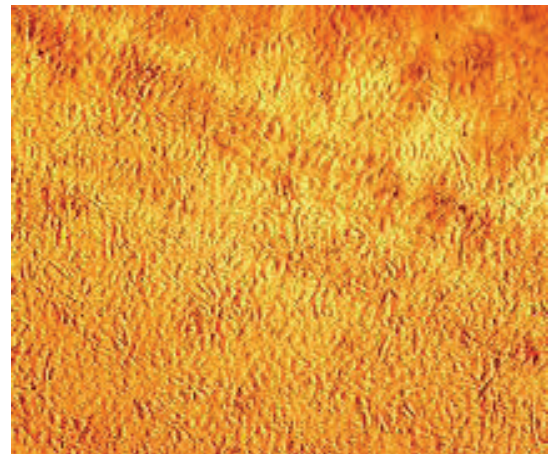

(b)

FIGURE 6: Fibroblast cells growth on the hydrogel sample (100×): (a) After 24 hours; (b) After 48 hours.

2.3. Antibacterial Analysis. A suspension of each four bacteria (S.epidermidis, S.aureus, P. aeruginosa, and E. coli K12) was prepared in broth from fresh colonies after overnight incubation, and the turbidity was adjusted to the $0.5 \mathrm{McFar}-$ land standard $\left(1.5 \times 10^{8}\right.$ c.f.u. $\left./ \mathrm{mL}\right)$. A part of this bacterial suspension $(10 \mathrm{~mL})$ was added to each vial containing the dressing. Control broths with bacterial inoculation were also included. The vials were then incubated with agitation at $35^{\circ} \mathrm{C}$ in a water bath. $10 \mathrm{~mL}$ of the bacterial broth were sampled from each vial at specific time intervals $(0,24 \mathrm{~h})$, and serial 10 -fold dilutions for each aliquot were prepared in broth. Duplicate aliquots $(25 \mathrm{~mL})$ of each of the serially diluted samples were spread on plates. The plates were then incubated overnight at $35^{\circ} \mathrm{C}$ and colonies counted (c.f.u./mL). The dilutions that allowed quantification (10150 colonies) were counted, and the mean counts calculated. vials, containing the antimicrobial dressings as well as the control dressing (Comfeel sample) together with the culture and the broth controls, were included in each experiment for each organism. Plate counts were measured in triplicate, and each experiment was repeated three times to obtain a mean value of c.f.u. counts. Results were evaluated by $K$ square statistical analysis $(P<0.05)$.

2.4. Cytotoxicity Analysis. The fibroblast cell suspension (L929) was prepared from the mouse tail. The surface of the
Comfeel sample (check) was well cleaned by using cotton and alcohol as well as the hydrogel samples. Pieces $(0.5$ $\times 0.5 \mathrm{~cm}$ ) of Comfeel and the main sample were cut and placed in one of the Petri dish wall (each one separately) by using a sterilized pincer. $3 \mathrm{cc}$ of the cell suspension was removed by pipette and poured on the Comfeel and the main samples. Then, all of the samples were placed in the Memmert incubator at $37^{\circ} \mathrm{C}$ for 24 and 48 hours, separately. The samples in the polystyrene Petri dish were removed from the incubator after 24 and 48 hours and were studied by Nikon Eclipse Ts-100 photonic microscope $(100 \times)$.

\section{Results and Discussion}

3.1. Antifungal Analysis. The results of the growth effect of Candida albicans fungi onto two hydrogel samples containing polyvinyl pyrrolidone, polyethylene glycol, agar, water, and the Comfeel as check sample are provided as follow.

The results in Table 1 have shown that fungi clearly grow onto the Comfeel surface (control sample), due to the lack of antifungal effect, but fungal growth is negative for hydrogel sample. Also, the results of the fungi growth on the check and hydrogel sample are shown in Figures 1 and 2, and they confirmed the results of Table 1. Figures $1(a)$ and 1(b) showed the bottom and the top surface of hydrogel sample 
TABLe 1: Antifungal analysis results.

\begin{tabular}{lllllllllll}
\hline \multirow{2}{*}{ Sample } & \multicolumn{10}{c}{ C. albicans } \\
& 1 & 2 & 3 & 4 & 5 & 6 & 7 & 8 & 9 & 10 \\
\hline Hydrogel sample & - & - & - & - & - & - & - & - & - & - \\
Comfeel sample & + & + & + & + & + & + & + & + & + & + \\
\hline
\end{tabular}

$K$ Square analysis: $P<0.05$.

effect on fungi growth, respectively. Also, Figures 2(a) and 2(b) showed the bottom and the top surface of the control sample effect on fungi growth, respectively, as well.

Figure 1 revealed the hydrogel sample with the lack of full growth of fungi on the surface of the hydrogel (Figure 1(a) showed only some fungi on the surface). Figures 2(a) and 2(b) demonstrated the full growth of fungi onto the surface of Comfeel (check sample).

3.2. Antibacterial Analysis. The bactericidal activities of the antimicrobial dressings against the S.epidermidis, S.aureus, $P$. aeruginosa, and $E$. coli $\mathrm{K} 12$ was indicated by a reduction in bacterial counts presented as $\log _{10}$ c.f.u. (colony forming units) $\mathrm{mL}^{-1}$ over time. The Results have shown that the hydrogel sample relative to the check sample was able to remove up to $30 \%$ of $S$. epidermidis, S. aureus, and E. coli K12 after 24 hours. But on P. aeruginosa, the situation was different; $P$. aeruginosa possess an increasing ability to resist antibacterial agent and Its intrinsically resistant to antimicrobial agents might be because of its low permeability of its cell wall and Its genetic capacity to express a wide repertoire of resistance mechanisms so no effect was detected on P. aeruginosa. Antibacterial test of two samples revealed a better biocompatibility of the hydrogel sample in comparison with Comfeel check sample that show in the Figure 3. Antibacterial test of two samples revealed a better biocompatibility of the hydrogel sample in comparison with Comfeel check sample.

3.3. Cytotoxicity Analysis. The cytotoxicity of hydrogel, Comfeel check sample and polystyrene control Petri dish were studied by fibroblast cells (1929) of mouse tail. Figure 4 showed the fibroblast cells growth on the polystyrene control Petri dish (TCPS). The growth of fibroblast cells on the Comfeel and hydrogel samples are shown in Figures 5 and 6.

Figures 4(a) and 4(b) clearly demonstrated the fibroblast cells growth on the polystyrene control Petri dish (TCPS). As shown in Figures 5 and 6, the fibroblast cells are well clung and grown on the check (Comfeel) and hydrogel samples did not significantly differ to control sample. These results have shown a proper biocompatibility and viability of the hydrogel sample.

\section{Conclusion}

In this study, the hydrogel biocompatibility was compared with Comfeel sample. In vitro biocompatibility assessments included cytotoxicity, antifungal, and antibacterial tests, which were the most important in vitro tests for bed sore wounds dressings. According to the observations of the cytotoxicity analysis, both of the hydrogel and the check samples showed good biocompatibility. It means that attachment or viability cells of both hydrogel and check sample are appropriate. The results of antibacterial test showed a rather better biocompatibility of the hydrogel sample than the Comfeel check sample. Also, observations of antifungal test demonstrated the lack of attachment and fungal growth on the surface of the hydrogel sample unlike the Comfeel check sample.

\section{References}

[1] S. Bale and D. Leaper, "Bandages and other therapies," in An Introduction to Wounds London, S. Bale, K. G. Harding, and D. Leaper, Eds., pp. 63-72, Emap Healthcare, 2000.

[2] M. Miller and G. Deborah, "The physiology of wound healing found in wound management theory and practice," in Nursing Times Book, pp. 14-20, 1999.

[3] M. Choucair and T. Phillips, "A review of wound healing and dressing materials," Skin and Aging, vol. 6, pp. 37-43, 1998.

[4] D. Chaloner and M. Fletcher, "Clinical trials: comparing dressings," Nursing standard, vol. 7, no. 7, pp. 7-9, 1992.

[5] G. C. Xakellis and E. A. Chrischilles, "Hydrocolloid versus saline-gauze dressings in treating pressure ulcers: a costeffectiveness analysis," Archives of Physical Medicine and Rehabilitation, vol. 73, no. 5, pp. 463-469, 1992.

[6] M. Flanagan and J. Fletcher, "Film as a temporary dressing," Journal of Wound Care, vol. 3, pp. 339-345, 1994.

[7] C. Williams, "Intrasite gel: a hydrogel dressing," British Journal of Nursing, vol. 3, no. 16, pp. 843-846, 1994.

[8] V. Jones, "Alginate dressings and diabetic foot lesions," The Diabetic Foot, vol. 2, no. 1, pp. 8-14, 1999.

[9] M. L. Shannon and B. Miller, "Evaluation of hydrocolloid dressings on healing of pressure ulcers in spinal cord injury patients," Decubitus, vol. 1, no. 1, pp. 42-46, 1988.

[10] S. Thomas and P. Hay, "Assessing the hydro affinity of hydrogel dressings," Journal of Wound Care, vol. 3, pp. 89-91, 1995.

[11] T. Young, C. Williams, M. Benbow, M. Collier, V. Banks, and H. Jones, "A study of two hydrogels used in the management of pressure sores," in Proceedings of the 6th European Conference on Advances in Wound Management, D. Leaper, G. Cherry, C. Dealey, J. Lawrence, and T. Turner, Eds., pp. 103-106, Macmillan Magazines Ltd, 1996.

[12] N. Hilmy, D. Darwis, and L. Hardiningsih, "Poly $(\mathrm{N}-$ vinylpyrrolidone) hydrogels: 2 . hydrogel composites as wound dressing for tropical environment," Radiation Physics and Chemistry, vol. 42, no. 4-6, pp. 911-914, 1993.

[13] Z. Ajji, G. Mirjalili, A. Alkhatab, and H. Dada, "Use of electron beam for the production of hydrogel dressings," Radiation Physics and Chemistry, vol. 77, no. 2, pp. 200-202, 2008. 

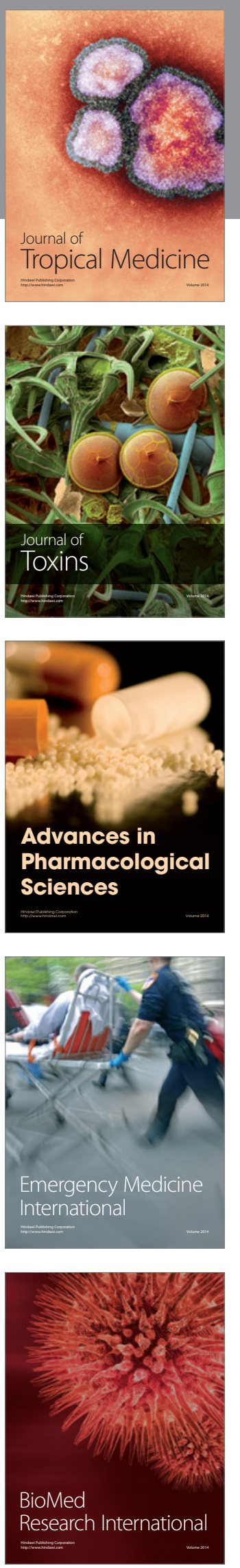
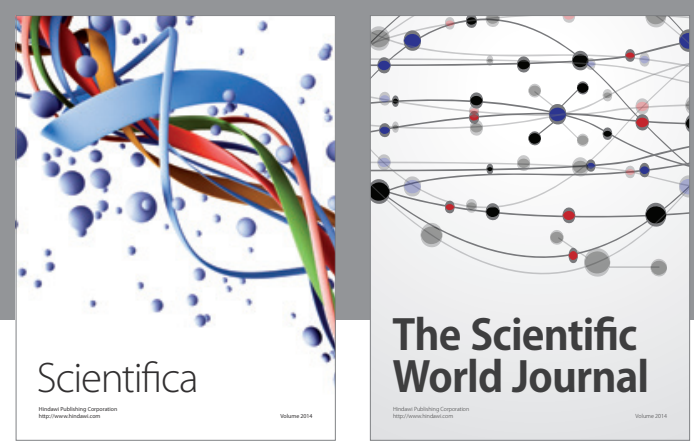

The Scientific World Journal
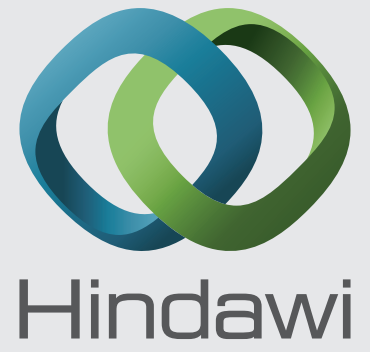

Submit your manuscripts at

http://www.hindawi.com
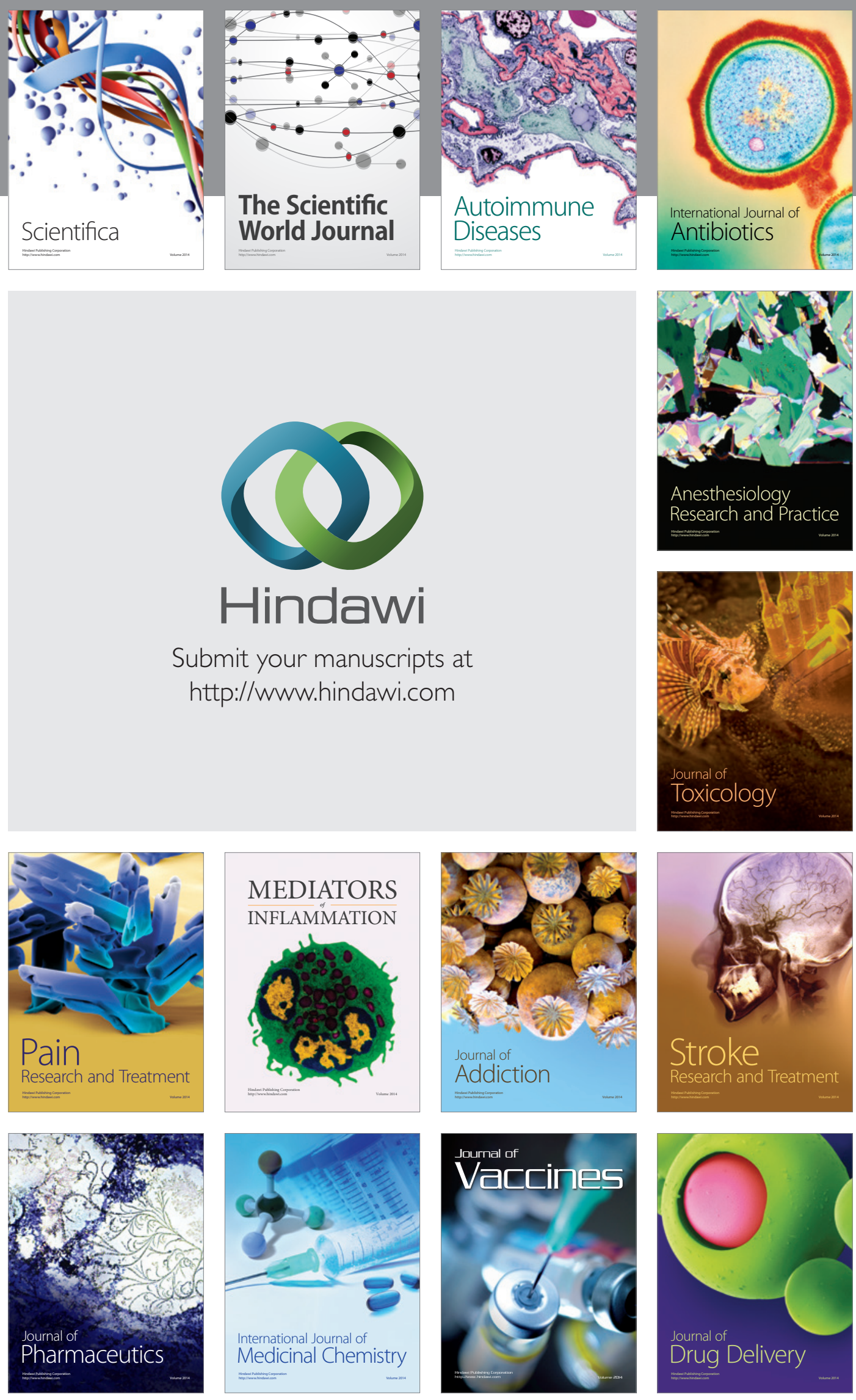